\title{
A brief review of some strange and unusual poisoning in children
}

\author{
Mohammad Hossein Kamaloddini, Anahita Alizadeh Ghamsari, Bita Dadpour* \\ Medical Toxicology Research Center, School of Medicine, Mashhad University of Medical Sciences, Mashhad, Iran
}

\begin{abstract}
Dear Editor
Acute pediatric poisoning is still considered as a universal health problem. This indicates a relatively common reason for children hospitalization in emergency units. Evidence shows an incidence rate of $0.33 \%$ to $7.6 \%$ for children poisoning (1) .

Children aged 1-5 years are at the highest risk for poisoning and this age range includes $80 \%$ of all pediatric poisoning cases (1). While household products and cleansers are the most responsible agents for poisonings in children aged 2-3 years, the medications left open or kept in the cupboard are the most common causes of poisoning in children aged 3 to 5 years (2)

Noticeably, only small amounts of some medications may lead to severe toxicity in children (3). For example; although toxic dose is estimated to be about $10-20 \mathrm{mg} / \mathrm{kg}$ for TCA (three cyclic anti-depressants), only one pill may cause severe toxicity or only one pill of drugs in Calcium channel blockers class may cause severe toxicity in children under three years of age. This also can be related to the severe salicylate toxicity following the consumption of even as much as a phalange of methyl salicylate gel in a toddler.

The Clinical Toxicology Center in Imam Reza hospital in Mashhad is the referral center of poisonings in the East of Iran and many cases of poisoned children are referred to this center and some cases are admitted.

In this letter we report some specific, strange and unusual poisonings in children admitted in our center.

Table 1 shows unusual and strange poisonings in
\end{abstract}

Received: 1 July 2019; Accepted: 25 September 2019;

Published online: 3 October 2019

*Corresponding author: Bita Dadpour,

Email: dadpourb@mums.ac.ir

Competing interests: None.

Funding information: There is none to be declared.

Citation: Kamaloddini MH, Alizadeh Ghamsari A, Dadpour B. A brief review of some strange and unusual poisoning in children. Journal of Emergency Practice and Trauma 2021, 7(1): 1-3. doi: 10.34172/ jept.2019.17. children. It is important to mention that this table can be updated over time. Physicians should be aware of these unusual condition sand management options that are recommended.

We should never use vacuum cleaners to clean mercury. The vacuuming will put mercury into the air and increase exposure. Never use a broom to clean up mercury. It will break the mercury into smaller droplets and spread them. Never pour mercury down a drain. After cleanup: Remember to keep the area well ventilated to the outside (i.e., open windows and turn on fans in the exterior windows) (4).

Authors' contributions

All of the authors participated in data collection, analysis and writing this article

Ethical Issues

This investigation was approved by the Ethics Committees of Mashhad University of Medical Sciences.

\section{References}

1. Sahin S, Carman KB, Dinleyici EC. Acute poisoning in children; data of a pediatric emergency unit. Iran J Pediatr 2011; 21(4): 479-84.

2. Mutlu M, Cansu A, Karakas T, Kalyoncu M, Erduran E. Pattern of pediatric poisoning in the east Karadeniz region between 2002-2006: increased suicide poisoning. Hum Exp Toxicol 2010; 29(2): 131-6. doi: 10.1177/0960327109357141.

3. Nelson LS, Hoffman RS, Howland MA, Lewin NA, Goldfrank LR, Smith SW. Goldfrank's Toxicologic Emergencies. 11th ed. McGraw-Hill Education; 2019.

4. United States Environmental Protection Agency (EPA). What to Do if a Mercury Thermometer Breaks. [cited 2019 Jun 25]; Available from: https://www.epa.gov/mercury/ what-do-if-mercury-thermometer-breaks. 
Table 1. Characteristic of some unusual poisonings in children

\begin{tabular}{|c|c|c|c|c|}
\hline Poisoning Group & $\begin{array}{l}\text { Name of poisonous } \\
\text { substance/drug }\end{array}$ & $\begin{array}{l}\text { Drug/substance compound / } \\
\text { Indication of use }\end{array}$ & $\begin{array}{l}\text { Toxidrome (main } \\
\text { clinical symptoms) }\end{array}$ & Management \\
\hline \multirow[t]{4}{*}{$\begin{array}{l}\text { Skin and hair care } \\
\text { products }\end{array}$} & Shampoo & $\begin{array}{l}\text { Sodium Lauryl Sulfate, sterol, } \\
\text { starch sulfate }\end{array}$ & Gl irritation & $\begin{array}{l}\text { There is no need for action except } \\
\text { carpet shampoo that is considered } \\
\text { caustic and the person should be } \\
\text { referred to a gastroenterologist. } \\
\text { For moderate to severe Irritant } \\
\text { effects of bathing shampoo } \\
\text { medical supportive treatment is } \\
\text { needed or refer the patient to a } \\
\text { gastroenterologist. }\end{array}$ \\
\hline & Hair conditioners & Bathing materials & Gl irritation and nausea & $\begin{array}{l}\text { Symptomatic treatment- if } \\
\text { symptoms are severe (acid and } \\
\text { alkali injury) }\end{array}$ \\
\hline & Soap & Bathing materials & Gl irritation and nausea & Symptomatic treatment \\
\hline & $\begin{array}{l}\text { Oxidizing liquid for } \\
\text { hair dye }\end{array}$ & $\mathrm{H} 2 \mathrm{O} 2$ & Gl irritation & Supportive measures \\
\hline \multirow{14}{*}{$\begin{array}{l}\text { Solvents and } \\
\text { House building } \\
\text { materials }\end{array}$} & $\begin{array}{l}\text { Absorbent materials } \\
\text { silaca gel }\end{array}$ & $\begin{array}{l}\text { Siliciom oxide-cobalt chloride } \\
\text { (blue)(rare) }\end{array}$ & Gl stimulation & $\begin{array}{l}\text { Preventing Gl irritation and inducing } \\
\text { vomiting }\end{array}$ \\
\hline & Instant drop glue & Hydrocarbon & & Cleansing from the tooth ${ }^{a}$ \\
\hline & Acetone & Nail polish cleaner & $\begin{array}{l}\text { Gl symptoms - } \\
\text { hyperglycemia }\end{array}$ & H2blocker for 7 to 10 days \\
\hline & $\begin{array}{l}\text { Plastic based paint } \\
\text { and oil based paint }\end{array}$ & Building painting. Hydrocarbon & $\begin{array}{l}\text { Gl stimulation -There are } \\
\text { considerable amounts of } \\
\text { lead in paints in Iran. }\end{array}$ & Supportive care- H2 Blocker/PPI \\
\hline & Oil paint thinner & Hydrocarbon & $\begin{array}{l}\text { Gastrointestinal } \\
\text { stimulation }\end{array}$ & Supportive care- H2 Blocker/PPI \\
\hline & Oil varnish & Hydrocarbon & $\begin{array}{l}\text { Gastrointestinal } \\
\text { stimulation }\end{array}$ & Supportive care- H2 Blocker/PPI \\
\hline & Silver oil & Hydrocarbon & Gl stimulation & Supportive care- H2 Blocker/ PPI \\
\hline & Polyester & Xylene (dimethylbenzene) & $\begin{array}{l}\text { Headache, fatigue, } \\
\text { Nausea, burning eyes } \\
\text { and nose }\end{array}$ & Supportive care- H2 Blocker/ PPI \\
\hline & Acetylene & Hydrocarbon & $\begin{array}{l}\text { Dizziness - headache - } \\
\text { mild epigastric pain }\end{array}$ & Similar to hydrocarbon poisoning \\
\hline & $\begin{array}{l}\text { Fluorine (1000 to } \\
5000 \text { PPM) and } \\
\text { hydrofluoric acid }\end{array}$ & $\begin{array}{l}\text { Industrial use and health care } \\
\text { material }\end{array}$ & $\begin{array}{l}\text { Severe lung and burn } \\
\text { injuries in the eye-ulcer } \\
\text { in the respiratory tract } \\
\text { Hypocalcemia and } \\
\text { hypomagnesemia }\end{array}$ & $\begin{array}{l}\text { Wash with plenty of water - rubbing } \\
\text { magnesium oxide - calcium } \\
\text { gluconate }\end{array}$ \\
\hline & Formaldehyde & $\begin{array}{l}\text { It is used in manufacture of resins } \\
\text { and textiles, as a disinfectant, } \\
\text { and as a laboratory fixative or } \\
\text { preservative. Formaldehyde } \\
\text { solution may contain methanol } \\
\text { in some degree }\end{array}$ & $\begin{array}{l}\text { Corneal burn- and } \\
\text { mouth burn nausea and } \\
\text { vomiting }\end{array}$ & $\begin{array}{l}\text { Rinse eyes and skin with plenty } \\
\text { of water-measures to deal with } \\
\text { methanol poisoning }\end{array}$ \\
\hline & $\begin{array}{l}\text { Potassium } \\
\text { polyacrylate }\end{array}$ & $\begin{array}{l}\text { Water absorbing materials in } \\
\text { agriculture }\end{array}$ & Nausea and vomiting & Supportive treatment \\
\hline & Building Gypsum & Chalk mixed with water & $\begin{array}{l}\text { Gl symptoms and } \\
\text { obstruction }\end{array}$ & $\begin{array}{l}\text { Surgical consultation and } \\
\text { supportive measures }\end{array}$ \\
\hline & Dead building plaster & Plaster wall & Gl stimulatory symptoms & Supportive measures \\
\hline & Dishwasher tablets & $\begin{array}{l}\text { Detergent ingredients. } \\
\text { Alkaline builders. Surfactants. } \\
\text { Chlorine compounds and } \\
\text { bleaching agents. Perfumes. } \\
\text { Alkaline salts and oxidizing } \\
\text { agents }\end{array}$ & Caustic injury & $\begin{array}{l}\text { Supportive care- High dose } \\
\text { H2 Blocker/PPI. Refer to a } \\
\text { gastroenterologist }\end{array}$ \\
\hline
\end{tabular}


Table 1. Continued

\begin{tabular}{|c|c|c|c|c|}
\hline Poisoning Group & $\begin{array}{l}\text { Name of poisonous } \\
\text { substance/drug }\end{array}$ & $\begin{array}{l}\text { Drug/substance compound / } \\
\text { Indication of use }\end{array}$ & $\begin{array}{l}\text { Toxidrome (main } \\
\text { clinical symptoms) }\end{array}$ & Management \\
\hline & Oven cleansers & $\begin{array}{l}\text { Water, sodium hydroxide/ } \\
\text { potassium hydroxide/lithium } \\
\text { hydroxide, surfactant, a } \\
\text { humectant }\end{array}$ & $\begin{array}{l}\text { Caustic injury (high } \\
\text { potent alkaline) }\end{array}$ & $\begin{array}{l}\text { Supportive care- High dose } \\
\text { H2 Blocker/PPI. Refer to a } \\
\text { gastroenterologist }\end{array}$ \\
\hline & Glassware cleansers & $\begin{array}{l}\text { Different composition but } \\
\text { mostly: Isopropyl alcohol, } \\
\text { Monoethanolamine, Butyl glycol }\end{array}$ & $\begin{array}{l}\text { Irritation, ketosis may } \\
\text { occur }\end{array}$ & Supportive care for Gl irritation \\
\hline & Hand sanitizer & $\begin{array}{l}\text { There are two main classes of } \\
\text { hand rubs: Alcohol based and } \\
\text { non-alcohol based. alcohol based } \\
\text { hand rubs are recommended } \\
\text { by WHO. Ethanol, isopropanol, } \\
\text { normal propylene, benzalkonium } \\
\text { chloride, skin softener and other } \\
\text { additives are ingredients of } \\
\text { Dermosept (a common hand } \\
\text { rub) }\end{array}$ & $\begin{array}{l}\text { Low blood sugar, coma } \\
\text { and seizure }\end{array}$ & $\begin{array}{l}\text { Alcohol poisoning management } \\
\text { (Airway protection, control of } \\
\text { hypoglycemia, control of seizure } \\
\text { and other supportive care (4) }\end{array}$ \\
\hline Plants & Anemone & & Delusions & Cancerous measures \\
\hline \multirow[t]{2}{*}{ Cosmetic products } & Perfume & $\begin{array}{l}\text { Fragrant essential oils or aroma } \\
\text { compounds, fixatives and } \\
\text { solvents }\end{array}$ & $\begin{array}{l}\text { Signs of toxicity of } \\
\text { ethanol and methanol }\end{array}$ & $\begin{array}{l}\text { Surface measurements and } \\
\text { appropriate therapeutic measures }\end{array}$ \\
\hline & Cosmetics & $\begin{array}{l}\text { Often lead free or contains small } \\
\text { amount }\end{array}$ & Stimulatory symptoms & Supportive measures \\
\hline \multirow[t]{7}{*}{ Miscellaneous } & $\begin{array}{l}\text { Mercury } \\
\text { thermometer }\end{array}$ & Elemental Mercury & Gl stimulatory symptoms & $\begin{array}{l}\text { If it is consumed orally no } \\
\text { further attempt is needed but it } \\
\text { recommended to Clean up mercury } \\
\text { spills }\end{array}$ \\
\hline & Watch battery & $\begin{array}{l}\text { Manganese dioxide - citric acid- } \\
\text { mercuric acid and zinc }\end{array}$ & Gastrointestinal irritation & $\begin{array}{l}\text { Chest and abdomen and internal } \\
\text { consultation for endoscopy }\end{array}$ \\
\hline & SIM card & Metal content- bond wire & Brief digestive symptoms & Supportive measures \\
\hline & Lady-bug & & Stimulatory symptoms & Supportive measures \\
\hline & Lizard & $\begin{array}{l}\text { Some kinds may include cyanide } \\
\text { contents in their body structure }\end{array}$ & Gl irritation & Supportive measures \\
\hline & $\begin{array}{l}\text { Sulfur match, } \\
\text { Wooden match or } \\
\text { match stick }\end{array}$ & Sulfur and phosphorus & Gastrointestinal irritation & Supportive measures \\
\hline & Firecracker & Gunpowder & $\begin{array}{l}\text { Mouth and stomach } \\
\text { ulcers }\end{array}$ & $\begin{array}{l}\text { Supportive measures: Treatment } \\
\text { similar to caustic injury } \\
\text { management if symptoms are } \\
\text { severe }\end{array}$ \\
\hline
\end{tabular}

a If drop glue causes the teeth to be stuck together, it should be cleansed with vinegar, and if it stuck to the fingers, it should be removed with acetone. If the lips are stuck or the mouth mucous membrane adheres, the patient should be NPO and TPN be initiated until the glue is gradually dissolved by saliva and the adhesion bands released. 